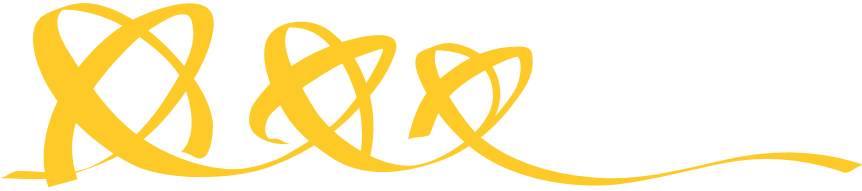 COMMUNICATIONS PHYSICS
}

EDITORIAL

\section{Credit where credit's due}

\author{
Recognition of the contribution made by reviewers to the publication of high quality research may take \\ many forms. We are passionate about ensuring quality in peer review and discuss here how to \\ acknowledge our reviewers fairly.
}

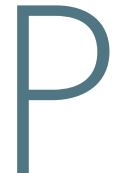
rompted by Peer Review Week, at Communications Physics we have been considering how we may best contribute to this year's theme of "Quality in Peer Review". Done well, peer review may be considered analogous to a positive feedback loop, in which reviewers play an indispensable role. This is because the quality of peer review authors receive depends intimately on that of the reports provided generously by our referees. In turn, the dialogue between authors and reviewers improves the final quality of our publications and provides valuable guidance to our editors. Hence, reviewers undoubtedly represent an essential resource for any peer reviewed journal. We believe it is vitally important to acknowledge the numerous reviewers who continue to provide high quality reports.

With this in mind, last year we publicly acknowledged some of our best referees, individuals who have risen over and above what is typically expected from a reviewer when providing guidance to our authors and editors. Furthermore, since March this year we have featured an outstanding referee, and in some cases a pair of co-reviewers, each month on our journal's webpage. In this way, we dedicate a space to directly highlighting the invaluable contribution the scientific community makes to producing publications of the highest possible quality and scientific integrity.

Earlier this year, we announced the introduction of Transparent Peer Review ${ }^{1}$, joining our sister journal, Nature Communications ${ }^{2,3}$, in offering authors the option to present anonymous and signed reviewer reports alongside their published manuscript. At present, Transparent Peer Review is in place for just over $40 \%$ of papers submitted to and published in Comms. Phys. in 2019. Readers may find the peer review file as a supplementary document, freely accessible on our webpage. It remains premature to reach a definitive verdict on how our community has responded to Transparent Peer Review, but the trends are encouraging. The value added to our published manuscripts during the peer review process is now visible to all. Furthermore, reviewers who choose to sign their reports gain visibility and recognition for their endeavours.

Intrinsically linked, however, to the desire to recognise and celebrate reviewers who provide high quality reports, is a concern regarding the pervasive practise of ghost-writing. Defined as the co-reviewing of a manuscript without providing a full list of contributors to the journal's editorial staff, ghost-writing is often conflated with the need to provide early career researchers (ECRs) with training in peer reviewing. At Communications Physics, we firmly believe it is important to decouple the valuable training experience gained by ECRs from the persistent practise of ghost-writing.

Earlier this year, a study blew away the cobwebs surrounding the issue of ghostwriting in the peer review process ${ }^{4}$. This global survey of almost 500 ECRs found that close to half of those questioned had received no credit for their contribution to reviewer reports and a further quarter did not know if their names appeared in the final version. These findings clearly 
indicate a breakdown in communication between ECRs, supervisors and editors. We find this insight concerning, as we seek to realise a more transparent peer review process and acknowledge those providing high quality reports for Communications Physics, regardless of their career stage.

The study, appearing in pre-print in April 2019, was the first to probe the complex motivations behind ghost-written reviewer reports. While half of respondents felt ghostreviewing to be unethical, over $70 \%$ confessed to making significant contributions to reviewer reports with no known credit. Thus, it is clear that while the majority of the community agrees with our belief that ghost-writing is unethical, the practise is entrenched in academic culture.

The peer review training received by ECRs from their supervisors is a common method by which this crucial skill is developed, second only to receiving reports on their own submitted manuscripts. Hence, the benefits of co-reviewing are clear. The authors of the recent study suggest that although ghost-writing of reports is considered ethically questionable, this behaviour is motivated by a second, strongly-held belief that only the invited reviewer should be seen to read or review the manuscript. It is undeniable that ghost-reviewing occurs, yet many seek to resolve the dichotomy between training and confidentiality by turning a blind eye. Thirdly, the community clings to the misconception that reports submitted by ECRs will be of lower quality.

As scientists driven by evidence and not opinion, we note this third belief is directly refuted by the study released earlier this year and preceding works. This data suggests there is no decrease in review quality when ECRs provide reports. Indeed, the study suggests many referee reports are already being provided by ECRs and so a substantial reduction in quality should not be expected. As a journal aiming to serve all members of the physics community, we seek to champion ECRs and when appropriate recognise their contribution to peer review in our Reviewer of the Month initiative. However, we are unable to do so if their contribution is not declared to our editors.

Only $4 \%$ of participants in the recent study cited the absence of a mechanism for reporting co-reviewers as the underlying cause of ghost-reviewing. Hence, the driving force behind ghost-written reviews is more complex. We acknowledge that some supervisors may not disclose the contribution of more junior colleagues in an effort to protect them from potential negative consequences at a vulnerable stage of their careers. While this sentiment is admirable, we wish to reassure reviewers that identities disclosed to our editors remain strictly confidential, unless the individual elects to sign their report. Furthermore, disclosing the contribution of ECRs allows us to diversify our pool of potential reviewers, leading to a higher quality peer review process.

Communications Physics asks for invited reviewers to disclose the names of any colleagues who contributed to their report to our editors privately. This is vital to maintain confidentiality for our authors, but also to ensure academic integrity. We believe in acknowledging the good work of our reviewers but, without full disclosure of authorship to our Editors, we cannot do so fairly and transparently. We are also signed-up to Publons, where reviewers may record their work and find recognition for their contributions. Lack of recognition for their work may affect both the career progression and visa or residency eligibility of $\mathrm{ECRs}^{4}$. Although not all ECRs would benefit equally from receiving public credit for their work, Communications Physics believe all contributors should be provided with the choice to do so.

A separate issue is the quality of training that is currently provided to ECRs. Most respondents reported handling only a few manuscripts during their academic careers, which are likely to be specific to their subfield. This is potentially an issue, since their training is restricted to a small number of individual experiences, occurring outside of an evidence-based framework. Furthermore, half of ECRs did not receive feedback from their supervisors on the contributions made to reports, leaving them with little potential for development. By publishing signed or anonymous reviewer reports, we seek to provide a library of reference materials across the breadth of physical disciplines and diverse reviewer styles. We hope this will broaden the experience of reviewers and, in turn, increase the quality of reports provided.
While the motivations behind ghostwriting in peer review are complex, often reinforced by cultural perceptions regarding the ownership of an ECR's work, a perceived breach of confidentiality or timepressure, we remain dedicated to providing a transparent and ethical review process for our authors. Acknowledging the significant contribution many ECRs make to peer review lifts the ethical dilemma regarding ghost-writing and promotes a more authentic scientific debate within the physics community. Both editors and academics may play a role in this process. At Communications Physics, we are committed to developing initiatives to ensure high quality peer review for our authors and recognising the valuable contributions made by those who review on our behalf.

Published online: 16 September 2019

\section{References}

1. Editorial. And we reached one at Communications Physics. Comms. Phys. 2, 19 (2019).

2. Editorial. Transparent peer review at Nature Communications. Nat. Commun. 6, 10277 (2015)

3. Editorial. Transparent peer review one year on. Nat. Commun. 7, 13626 (2016).

4. McDowell, G. S., Knutsen, J., Oelker, S. K. \& Lijek, R. S. Co-reviewing and ghostwriting by early career researchers in the peer review of manuscripts. Preprint at bioRxiv https://doi.org/ $10.1101 / 617373$ (2019)

\section{(i)}

Open Access This article is licensed under a Creative Commons Attribution 4.0 International License, which permits use, sharing, adaptation, distribution and reproduction in any medium or format, as long as you give appropriate credit to the original author(s) and the source, provide a link to the Creative Commons license, and indicate if changes were made. The images or other third party material in this article are included in the article's Creative Commons license, unless indicated otherwise in a credit line to the material. If material is not included in the article's Creative Commons license and your intended use is not permitted by statutory regulation or exceeds the permitted use, you will need to obtain permission directly from the copyright holder. To view a copy of this license, visit http:// creativecommons.org/licenses/by/4.0/.

() Springer Nature Limited 2019 